\title{
Comparison of Job Satisfaction and Job Stress Among Nurses, Operating Room and Anesthesia Staff
}

\author{
Ramin Rahmani $^{1}$ (D), Ali Ebrazeh², Farzad Zandi ${ }^{3}$, Roghayeh Rouhi ${ }^{4}$, Shirdel Zandi* ${ }^{*}$
}

1. MSc Student, Department of Occupational Hygiene Engineering, Student Research Committee, Hamadan University of Medical Sciences, Hamadan, Iran

2. Assistant Professor, Department of Public Health, Faculty of Health, Qom University of Medical Sciences, Qom, Iran

3. Assistant Professor, Department of Psychology, Qorveh Branch, Islamic Azad University, Qorveh, Iran

4. Bachelor of Nursing, School of Medicine, Zabol University of Medical Sciences, Zabol, Iran

5. MSc Student, Student Research Committee, Hamadan University of Medical Sciences, Hamadan, Iran

\begin{tabular}{ll}
\hline Article Info & ABSTRACT \\
\end{tabular}

Received: 2020/11/14;

Accepted: 2020/12/28;

ePublished: $2020 / 12 / 28$

doi) 10.30699/jergon.8.4.103

Use your device to scan and read the article online

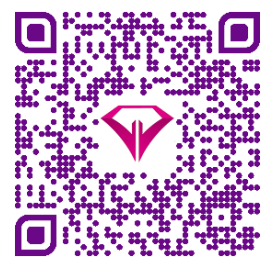

Corresponding Author Shirdel Zandi

MSc Student, Student Research Committee, Hamadan University of Medical Sciences, Hamadan, Iran

Email:

shirdel.zandi1994@yahoo.com
Background and Objectives: Paying attention to human resources is one of the basic principles for increasing productivity and quality of services in hospitals. Therefore, the present study was designed to compare the level of job satisfaction and job stress, and the association between these two components in nurses, operating room, and anesthesia staff.

Methods: This is a descriptive causal-comparative study, performed in December 2019 in Zabol hospitals with the participation of 175 nurses, operating room, and anesthesia staff. A three-part questionnaire consisting of demographic information, job satisfaction, and job stress was used to collect data. Then analysis of data was performed using SPSS 20 and the Pearson correlation coefficient, independent t-test, and ANOVA.

Results: In this study, it was found that nursing group had the highest job satisfaction and anesthesia and operating room staff had the lower job satisfaction. Also, the highest and lowest levels of job stress were related to anesthesiologists and nurses, respectively, and the differences in both variables were significant between the three groups. Moreover, the results showed that there was a significant negative correlation between job satisfaction and job stress, totally.

Conclusion: Due to that anesthesia staff was in a more unfavorable situation than others. We recommend that management planning and policies in hospitals be done by occupational groups and design and carry out the studies to assess the needs for each group.

Keywords: Job satisfaction, Job stress, Nursing group, Operating room, Anesthesia

Copyright (C) 2021, This is an original open-access article distributed under the terms of the Creative Commons Attribution-noncommercial 4.0 International License which permits copy and redistribute of the material just in noncommercial usages with proper citation.

\section{How to Cite This Article:}

Rahmani R, Ebrazeh A, Zandi F, Rouhi R, Zandi SH. Comparison of Job Satisfaction and Job Stress Among Nurses, Operating Room and Anesthesia Staff. Iran J Ergon. 2020; 8 (4) :103-114. 


\section{Extended Abstract}

\section{Introduction}

Paying attention to human resources is one of the basic principles for increasing productivity and quality of services in hospitals. Therefore, the present study was designed to compare the level of job satisfaction and job stress, and the association between these two components in nurses, operating room, and anesthesia staff.

\section{Methods}

This is a descriptive causal-comparative study, performed in December 2019 in Zabol hospitals with the participation of 175 nurses, operating room, and anesthesia staff. A three-part questionnaire consisting of demographic information, job satisfaction, and job stress was used to collect data.

The analysis of the study data consisted of two parts: the first part, a descriptive of the results using descriptive statistical techniques (tables and figures and central indexes and scattering including mean and standard deviation). The second part was inferential analysis using t-test and one-way analysis of variance and Pearson correlation coefficient. All these analyses were performed using SPSS 20 (SPSS Inc., Chicago, IL., USA) and the significance rate was considered 0.05 .

\section{Results}

In this study, it was found that nursing group had the highest job satisfaction and anesthesia and operating room staff had the lower job satisfaction. Also, the highest and lowest levels of job stress were related to anesthesiologists and nurses, respectively, and the differences in both variables were significant between the three groups. Moreover, the results showed that there was a significant negative correlation between job satisfaction and job stress, totally.

Table 1. Demographic and occupational information in the studied population

\begin{tabular}{|c|c|c|c|c|c|}
\hline \multicolumn{2}{|c|}{ Variable } & $\begin{array}{c}\text { Nurse }(n=76) \\
\text { N }(\%)\end{array}$ & $\begin{array}{c}\text { OR staff }(n=61) \\
N(\%)\end{array}$ & $\begin{array}{c}\text { Anesthesia }(\mathrm{n}=\mathbf{3 8}) \\
\mathrm{N}(\%)\end{array}$ & P-value \\
\hline \multirow{2}{*}{ Gender } & Male & $28(36.8)$ & $24(39.3)$ & $12(31.6)$ & \multirow{2}{*}{0.736} \\
\hline & Female & $48(63.2)$ & $37(60.7)$ & $26(68.4)$ & \\
\hline \multirow{3}{*}{ Education } & Associate's degree & $19(25)$ & $16(26.3)$ & $10(26.3)$ & \multirow{3}{*}{0.387} \\
\hline & Bachelor's degree & $50(65.8)$ & $44(72.1)$ & $24(63.2)$ & \\
\hline & Master's degree & $7(9.2)$ & $1(1.6)$ & $4(10.5)$ & \\
\hline \multirow{2}{*}{ Job status } & Official & $49(64.5)$ & $42(68.9)$ & $27(71)$ & \multirow{2}{*}{0.746} \\
\hline & Project & $27(35.5)$ & $19(31.1)$ & $11(29)$ & \\
\hline \multirow{2}{*}{ Marital status } & Married & $58(76.3)$ & $40(65.6)$ & $25(65.8)$ & \multirow{2}{*}{0.310} \\
\hline & Single & $18(23.7)$ & $21(34.4)$ & $13(34.2)$ & \\
\hline & & M \pm SD & M \pm SD & M $\pm S D$ & \\
\hline \multicolumn{2}{|c|}{ Age } & $4.57 \pm 33.78$ & $7.09 \pm 33.80$ & $5.20 \pm 30.80$ & 0.099 \\
\hline \multicolumn{2}{|c|}{ Work history } & $4.33 \pm 8.82$ & $6.66 \pm 8.58$ & $5.05 \pm 6.95$ & 0.391 \\
\hline
\end{tabular}


Table 2. Results of one-way analysis of variance test to compare job stress and job satisfaction in the studied groups

\begin{tabular}{|c|c|c|c|c|c|c|}
\hline Variable & Change sources & $\begin{array}{c}\text { Total } \\
\text { squares }\end{array}$ & $\begin{array}{c}\text { Freedom } \\
\text { degrees }\end{array}$ & Mean squares & f & P-value \\
\hline \multirow{3}{*}{$\begin{array}{c}\text { Job } \\
\text { satisfaction }\end{array}$} & Intragroup & 2141.422 & 2 & 1070.711 & 3.311 & 0.039 \\
\hline & Intergroup & 55623.610 & 172 & 323.393 & & \\
\hline & Total & 57765.032 & 174 & & & \\
\hline \multirow{3}{*}{ Job stress } & Intragroup & 2428.686 & 2 & 1214.343 & 3.911 & 0.022 \\
\hline & Intergroup & 53398.207 & 172 & 310.455 & & \\
\hline & Total & 55826.894 & 174 & & & \\
\hline
\end{tabular}

Table 3. Pearson correlation values between job satisfaction and job stress by study groups

\begin{tabular}{|c|c|c|c|c|}
\hline Var & & Job satisfaction & Job stress & P-value \\
\hline \multirow{2}{*}{ Nursing } & $\begin{array}{c}\text { Job } \\
\text { satisfaction }\end{array}$ & 1 & 0.603 & \multirow{2}{*}{$0.001<$} \\
\hline & Job stress & 0.603 & 1 & \\
\hline \multirow{2}{*}{ OR staff } & $\begin{array}{c}\text { Job } \\
\text { satisfaction }\end{array}$ & 1 & 0.372 & \multirow{2}{*}{0.003} \\
\hline & Job stress & 0.372 & 1 & \\
\hline \multirow{2}{*}{ Anesthesia } & $\begin{array}{c}\text { Job } \\
\text { satisfaction }\end{array}$ & 1 & 0.577 & \multirow{2}{*}{$0.001<$} \\
\hline & Job stress & 0.577 & 1 & \\
\hline
\end{tabular}

\section{Discussion}

Based on the findings of this study, in the field of job satisfaction, it was found that nurses had the highest level of job satisfaction and operating room staff compared to anesthesia staff. The results of a study by Debara et al. [26] showed that the job satisfaction of operating room staff was higher than that of anesthesia staff and was consistent with the findings of the present study. However, based on studies by Sadeghi et al. [27] and Koshy et al. [28], it was found that the job satisfaction of anesthesia staff is higher than that of operating room staff. Also, based on the findings of the study of Borys et al., there was no significant difference between job satisfaction of operating room staff and nurses working in the ICU [9]. These discrepancies may be due to differences in employment status and factors affecting job satisfaction such as salaries, benefits, workload and employee relations [27].
According to many researchers, including Stewart [21], various factors are effective in employee job satisfaction, some of which are personal characteristics as internal factors and others such as organizational structure and culture, individual perception of workplace status and job security, relationships between coworkers and the relationship with the supervisor or superior are among the external factors affecting job satisfaction.

Another key finding of the present study was the comparison of job stress levels in the studied groups. In this regard, the results showed that the lowest and highest levels of job stress were related to nurses and anesthesia staff, respectively. One of the main reasons that anesthesia staff experience more stress compared to other study subjects may be the sensitivity of the work situation associated with anesthetizing 
patients and the risks of anesthesia for patients with certain conditions.

Another important finding of this study was to investigate the relationship between job stress and job satisfaction. In this regard, the results of Pearson correlation coefficient showed that there was a negative and moderate correlation between these two organizational components in terms of intensity, which was also statistically significant $(P<0.01)$. This result is quite consistent with studies conducted by Angermayer et al. [31] and Ghaseminezhad et al. [32]. Better yet, the more job satisfaction people have, the less job stress they will experience, or it can be inferred that people with less job stress will be more satisfied with their job. Also, based on the study of Batram et al. [33], it was found that there is an inverse and significant relationship between some dimensions of job stress and job satisfaction in nurses.

\section{Conclusion}

Due to that anesthesia staff was in a more unfavorable situation than others. We recommend that management planning and policies in hospitals be done by occupational groups and design and carry out the studies to assess the needs for each group.

\section{Acknowledgement}

The present study is the result of a student research project that has been approved by the ethics committee of Hamadan University of Medical Sciences with the code: ID IR.UMSHA.REC.1398.641 and has been supported by the Vice Chancellor for Research and Technology of this university. The authors of this article consider it necessary to thank the Vice Chancellor for Research of the University, especially Mr. Morteza Shamsizadeh, the honorable head of the Student Research Committee, and Dr. Mohammad Babamiri for their useful guidance.

\section{Conflict of Interest}

The authors declared no conflict of interest. 
مقاله يزوهشى

مقايسةُ رضايت شغلى و استرس شغلى در يرستاران و كاركنان اتاق عمل و هوشبَرى

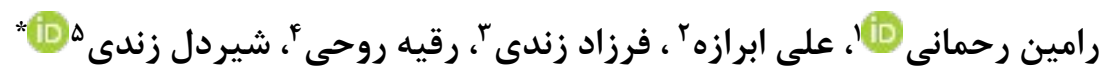

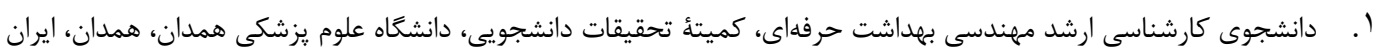

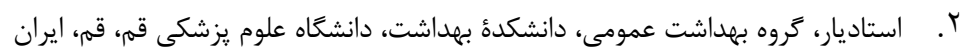

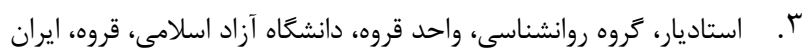

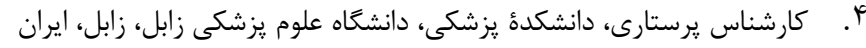

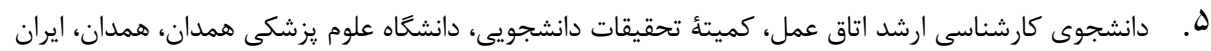

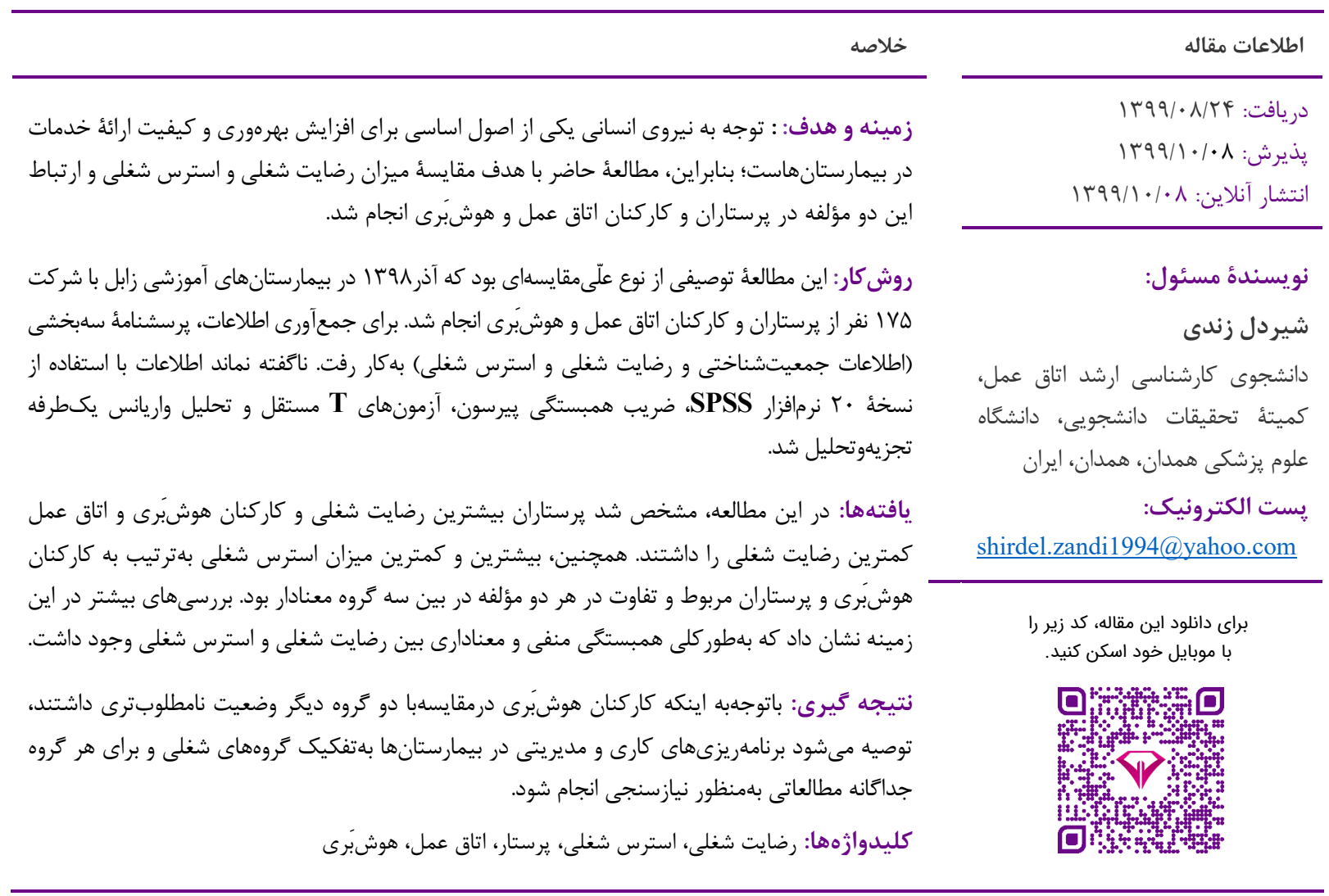

مقدمه

به سلامتى نيروى كارى بهعنوان يكى از ركنهاى اساسى هر

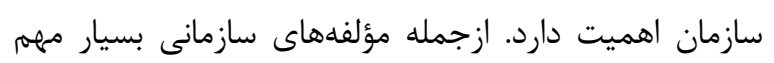
توجه به رضايت شغلى افراد است. درواقع، رضايت شغلى بيانكر أهريت

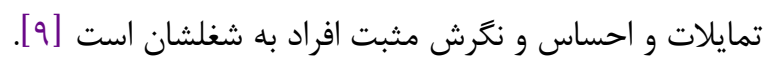
موضوع رضايت شغلى در سازمانها را از دو منظر مىتوان بررسى كرد: يكى بهخاطر احترام به انسان و تمايلاتش و ديخرى

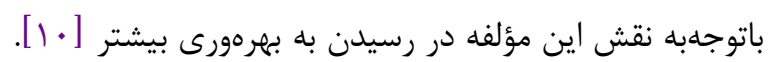

بررسىهاى يزوهشگ ان در محيطهاى كارى مختلف حاكى

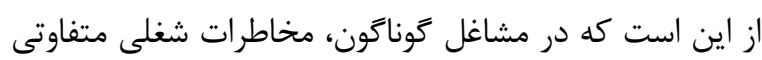
وجود دارد كه با عنوان عوامل زيانآور شغلى شناخته مىشوند و سلامتى شاغلان را تهديد ميىكند [1 تا هان]. دراينميان، دستهاى از اين مخاطرات بُعد روانشناختى افراد را درگير

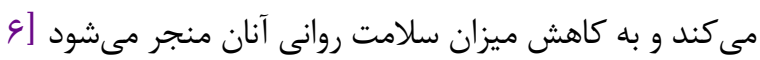
-1]. اين در حالى است كه سياست و خطِمشى هر سازمان بايد رسيدن به بيشترين ميزان بهرهورى باشد. در اين زمينه، توجه 
مؤلفه و ارتباط بين آنها در اين افراد، توصيف بهترى از شرايط شغلى آنان مهيا شود.

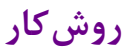

\section{1. امعه و نمونهُ هدف}

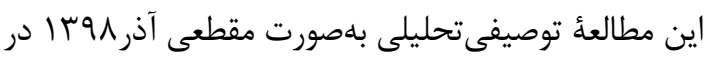

بيمارستانهاى آموزشى سطح شهر زابل انجام شد. جامعأ يزوهش شامل تمامى يرستاران بخش و كاركنان اتاق عمل و و

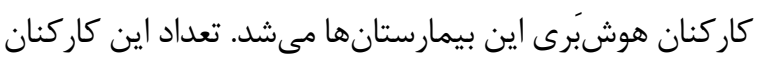

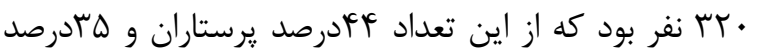
كاركنان اتاق عمل و آكرصد كاركنان هوشبَرى بودند. باتوجلهبه حجم جامعه و براساس جدول موركان، تعداد نمونهها

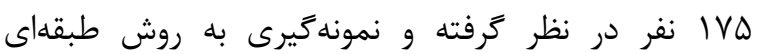
سهميهاى به نسبت يرستاران و كاركنان اتاق عمل و كاركنان هوشبَرى انجام شد. قبل از شروع مراحل كار، از افران

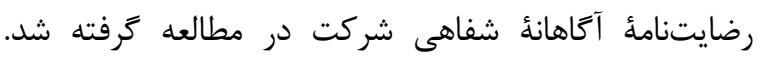
معيارهاى ورود به مطالعه عبارت بودند از: داشتن حداقل يك دافي سال سابقء كار بالينى و نداشتن اختلالات روانشناختى

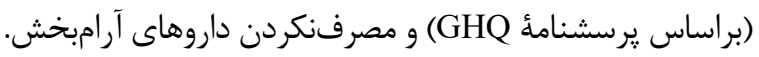

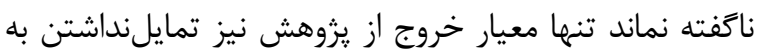

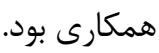

\section{ك. ابزارهاى جمع آورى دادهها}

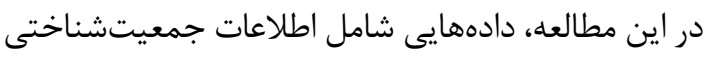
و شغلى (جنسيت، سن، سابقء كار، ميزان تحصيلات و...) و وضعيت رضايت شغلى و استرس بلصورت يرسشنامهاى و و

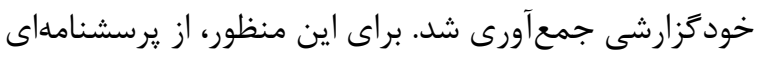

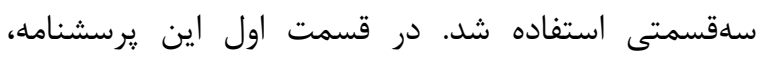
يرسشهايى دربارؤ ويزگى هاى فردى و شغلى و در قسمت دوم، سؤالات يرسشنامة استرس شغلى درئل

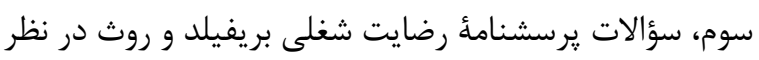

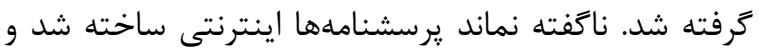
دراختيار شركت كنندكان قرار كرفت.

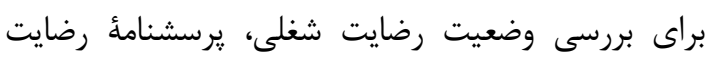

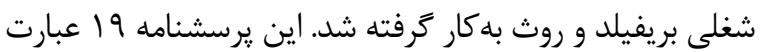

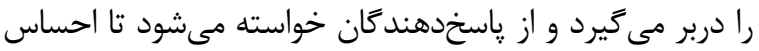

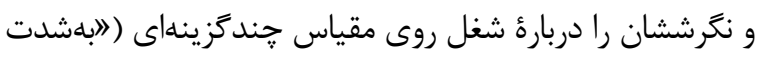

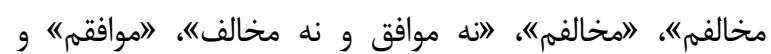
"بهشدت موافقم") مشخص كنند. نمرهذارى نيز بدينترتيب
اين مؤلفه از عوامل مختلفى تأثير مىيذيرد كه از همه مهمتر

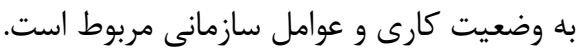

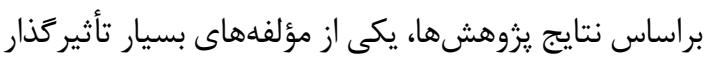

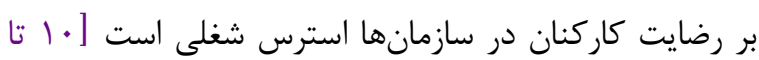

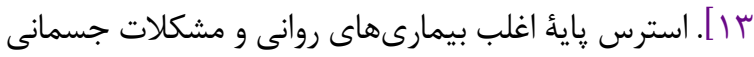

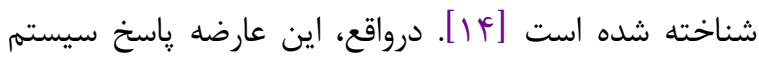

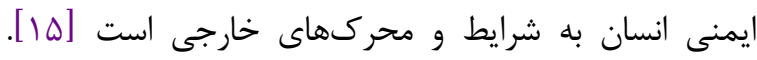

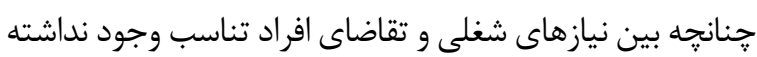
باشد يا افراد در محيط كارىشان با مشكلاتى مثل ابهام در

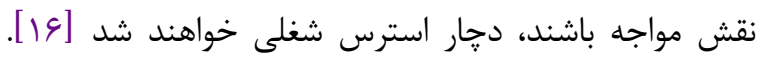

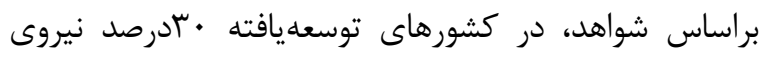
كارى به استرس مبتلا هستند و اين ميزان در كشورهاى

درحالتوسعه حتى بيشتر است [هارئه

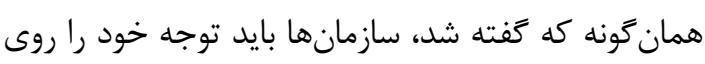

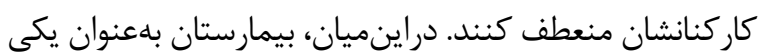
از سازمانهاى حياتى توجه بيشترى مى طلبد؛ حراكه بلطور

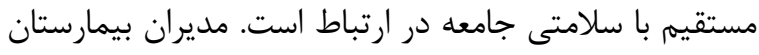

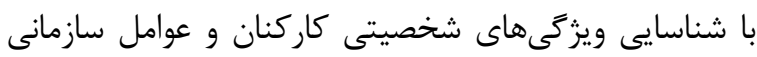
مؤثر بر اين دو مؤلفه، يعنى رضايت شغلى و استرس شغلى

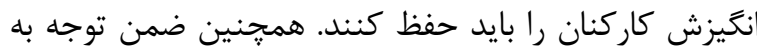

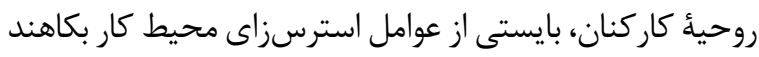
تا افراد در بهينهترين حالت ممكن خدمات و وظايفشان ران را انجام

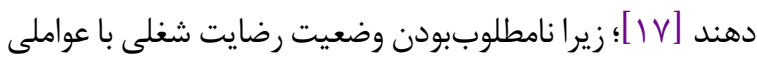
همجُون غيبت از كار و كاهش كيفيت كارى كاركنان در ارتباط

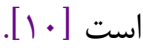

براساس مطالعات بيشين، استرس و رضايت شغلى هركدام

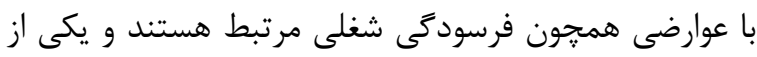

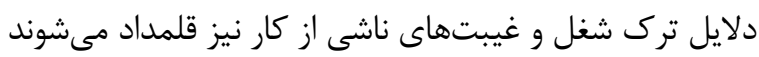

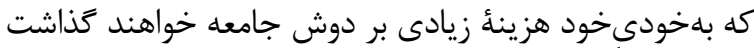

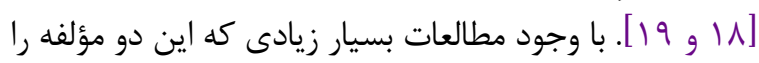

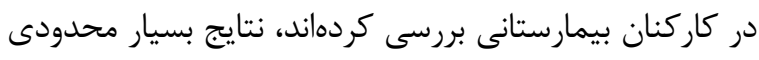

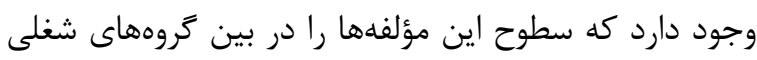

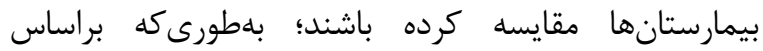
جستوجوى محققان، تاكنون مطالعهاى در داخل كشور در اين

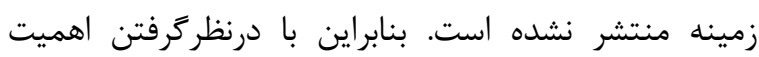

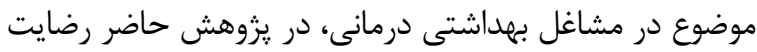

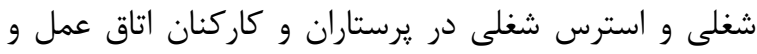

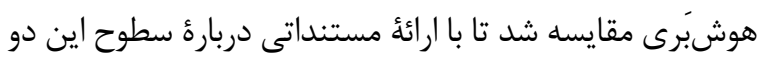


در شكل ا، توصيفى از وضعيت رضايت شغلى و استرس شغلى در سه كروه ارائه شده است. دربارهٔ رضايت شغلى بايد

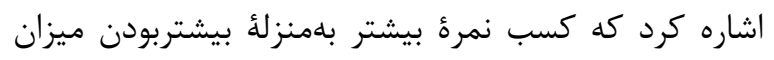

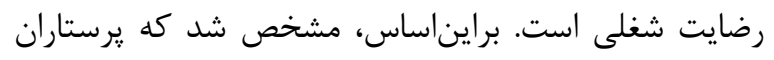

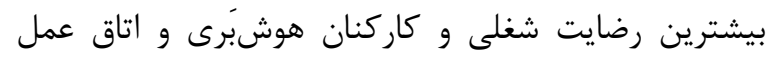
كمترين رضايت شغلى را داشتند. همجنينين، باتوجلوبه اينكه

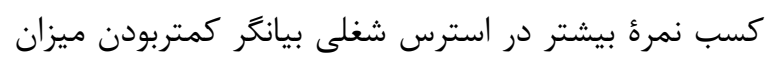

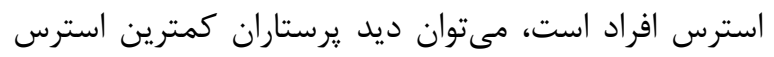

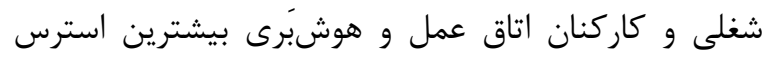
شغلى را تجربه مىكردند. بلمنظور مقايسٔ رضايت شغلى و استرس شغلى درد در سه

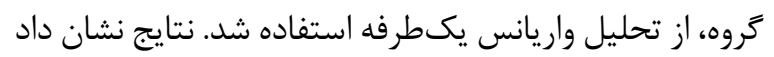

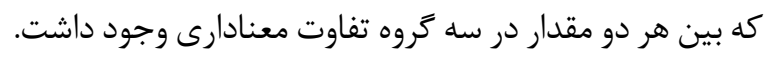

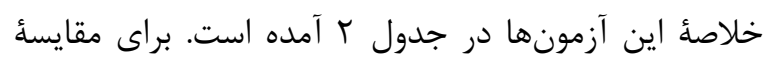

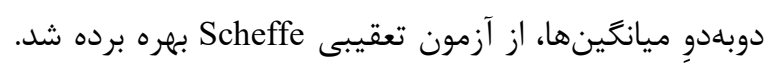

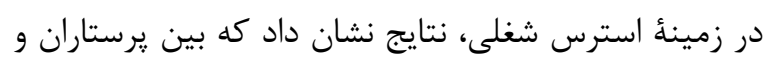

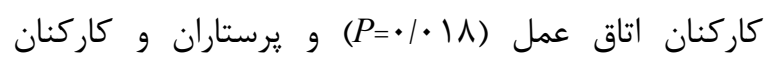

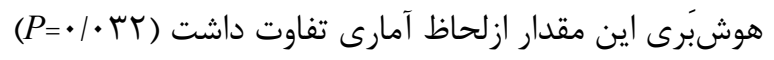

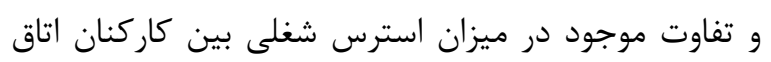

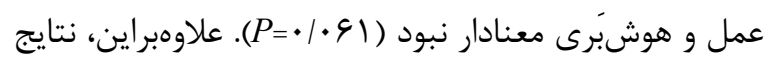

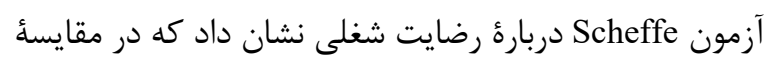

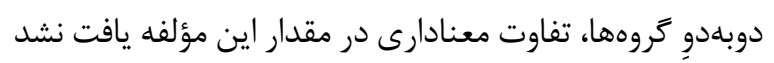
.$(P>\cdot / \cdot \Delta)$

يكى از اهداف يزوهش حاضر، بررسى ارتباط بين استرس

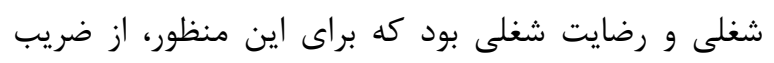

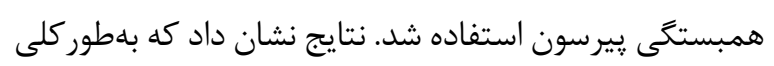

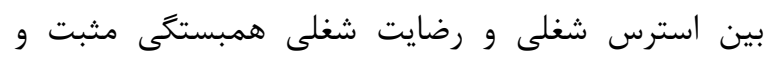

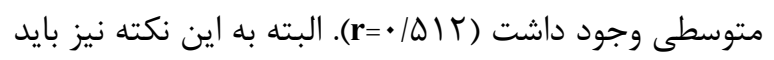

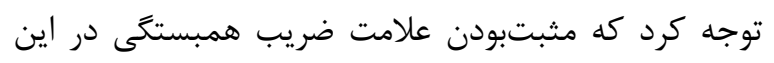

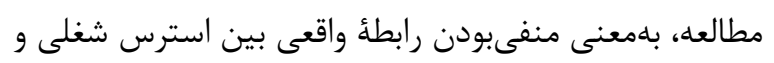

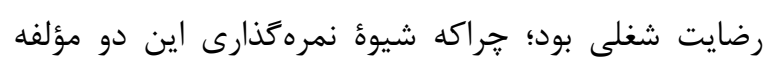
برعكس هم است.

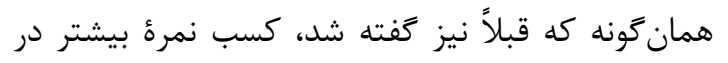

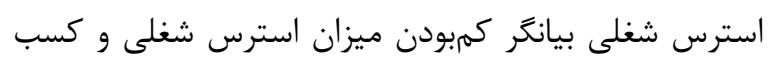

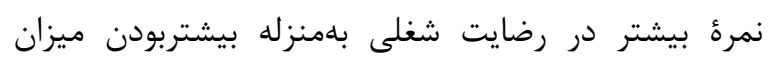

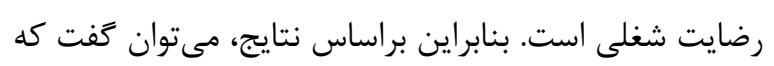

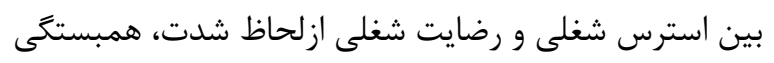

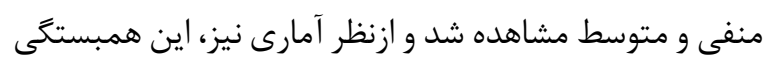

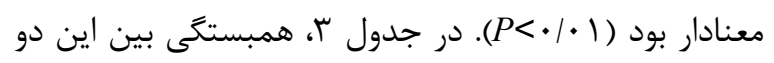

است كه به ززينهٔ لبهشدت مخالفمه كمترين امتياز و به كزينهٔ

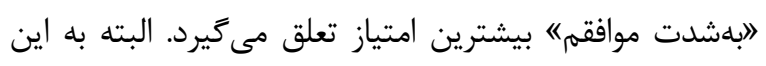

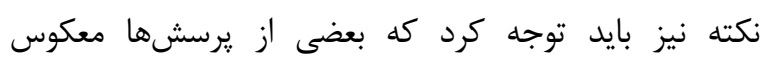

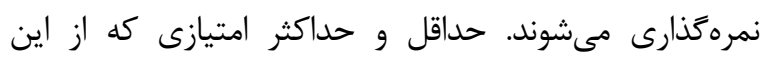

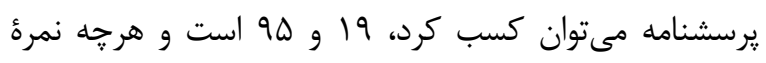

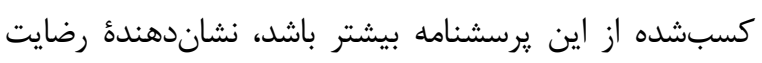

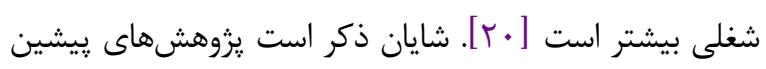

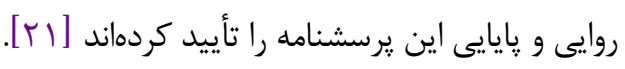
بلمنظور ارزيابى وضعيت استرس شغلى برسئ در كروههاى

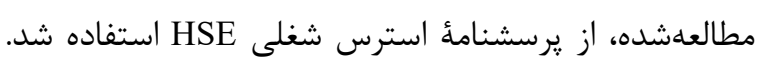

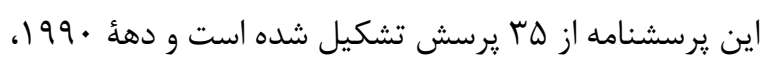

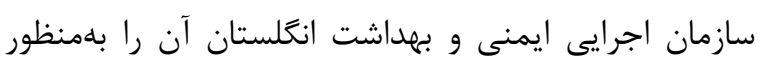

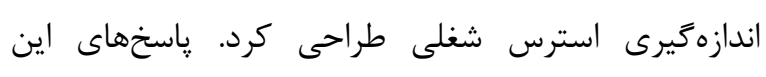

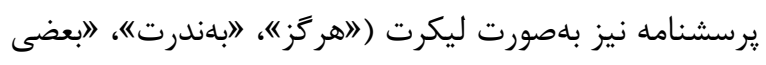
اوقات)، ااغلب" و "همواره) است كه بلهترتيب از يكى تا ينج

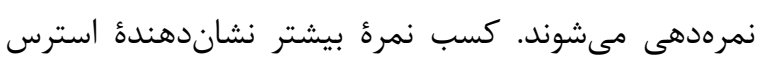

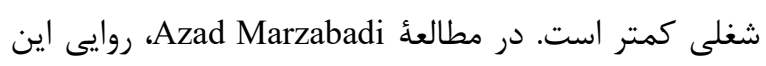

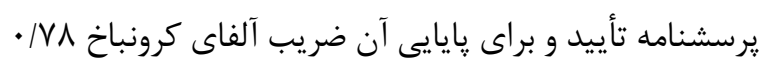

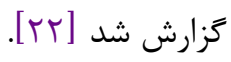

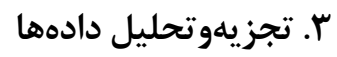

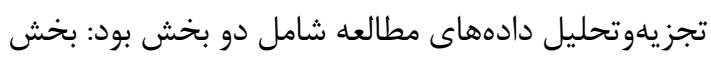

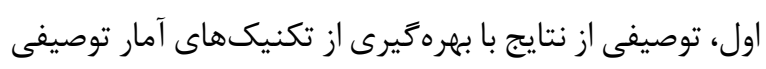

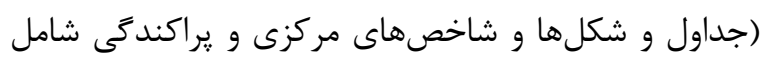

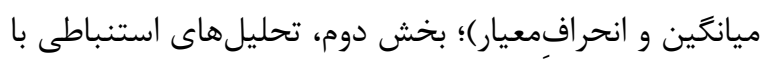

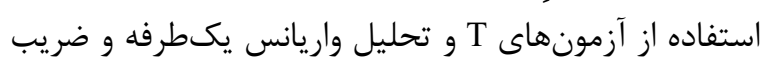

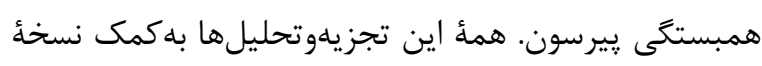

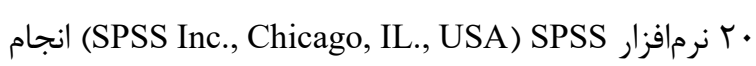

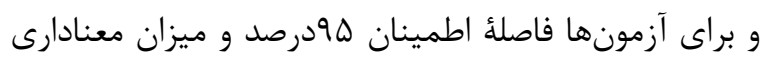

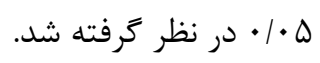

بافته ها

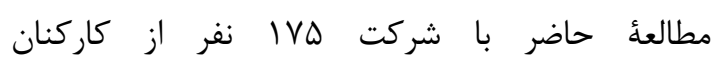

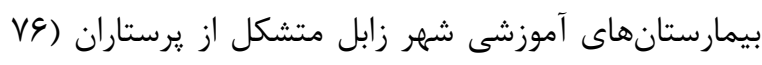

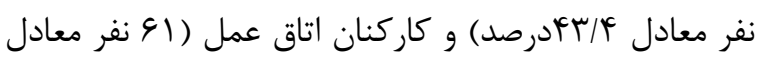

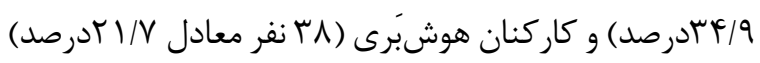

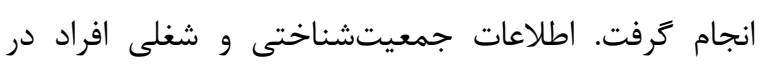

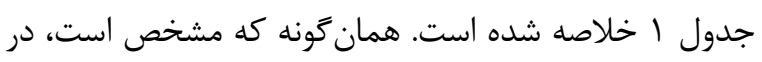

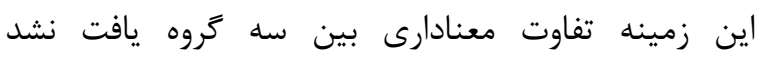

.$(P>\cdot / \cdot \Delta)$ 


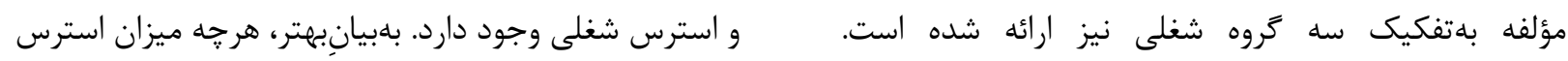

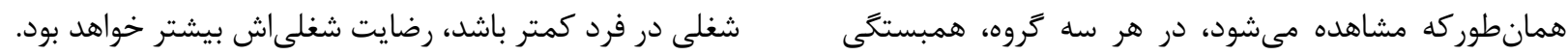

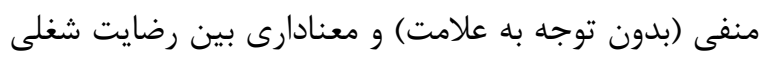

جدول ا. اطلاعات جمعيتشناختى و شغلى در جمعيت مطالعهشده

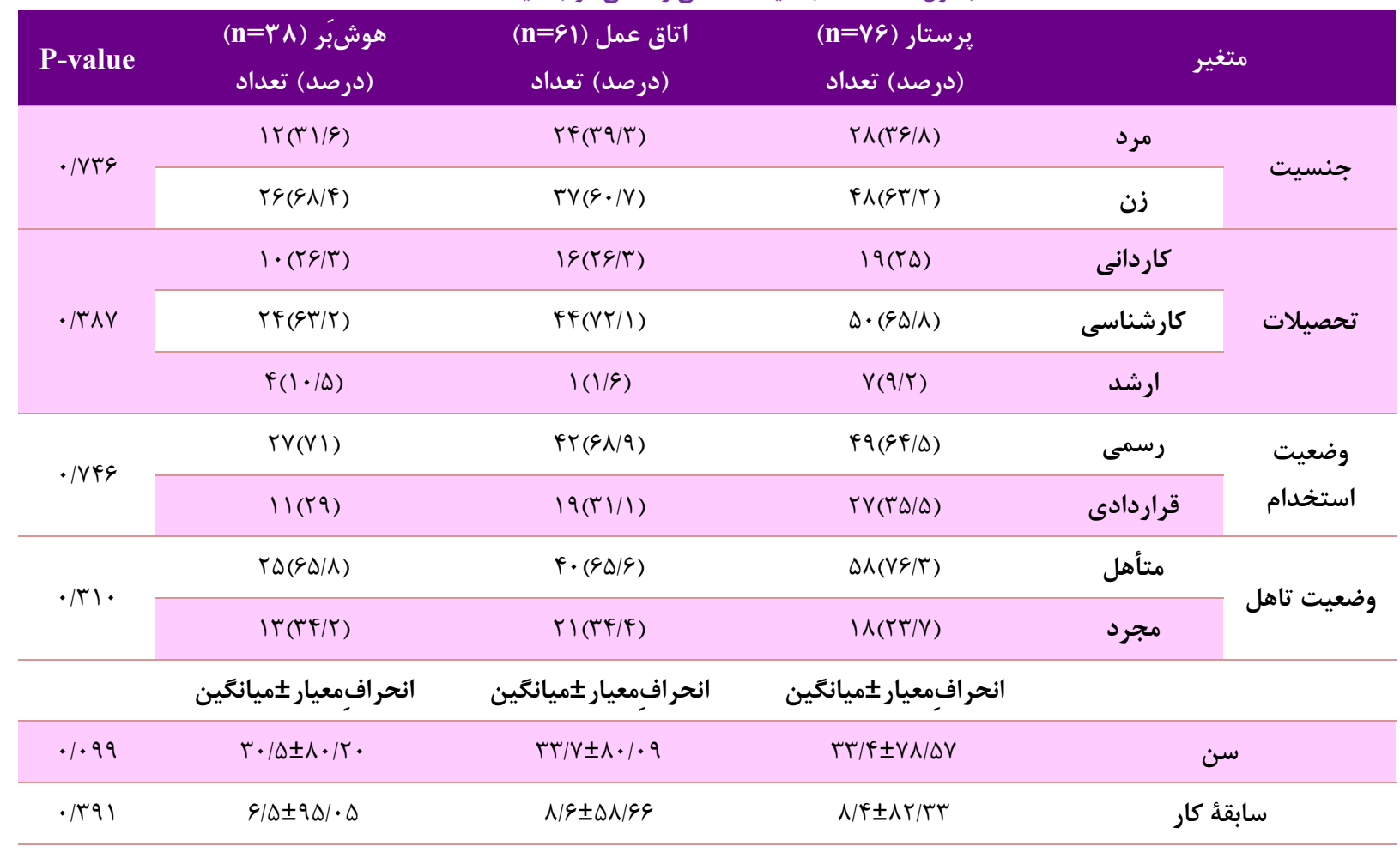

جدول r. نتايج آزمون تحليل واريانس يكىطرفه براى مقايسةُ استرس شغلى و رضايت شغلى در گروههاى مطالعهشده

\begin{tabular}{|c|c|c|c|c|c|c|}
\hline p-value & مقدار f & ميانََين مجذورات & درجات آزادى & مجذوروات مجموع & منابع تغيير & متغير \\
\hline \multirow[t]{3}{*}{. } & & $1 \cdot V \cdot / V 11$ & r & TIFI/ETT & بين گروهى & \multirow{3}{*}{ رضايت شغلى } \\
\hline & & rrmprr & IVT & $\Delta \Delta G K M|G|$. & درون گروهى & \\
\hline & & & IVF & $\Delta V V G \Delta / \cdot T T$ & كل & \\
\hline \multirow[t]{3}{*}{. $/ . r T$} & $r / 911$ & $|r| F / r F r$ & r & TYYN/GAG & بين گروهى & \multirow{3}{*}{ شغلى استرس } \\
\hline & & $m \cdot / F \Delta \Delta$ & IVT & $\Delta r r q \Lambda / T \cdot V$ & درون گروهى & \\
\hline & & & IVF & $\Delta \Delta \wedge T \& / \Lambda q T$ & كل & \\
\hline
\end{tabular}

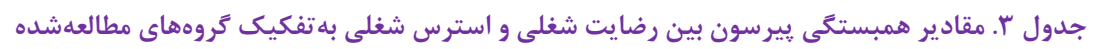

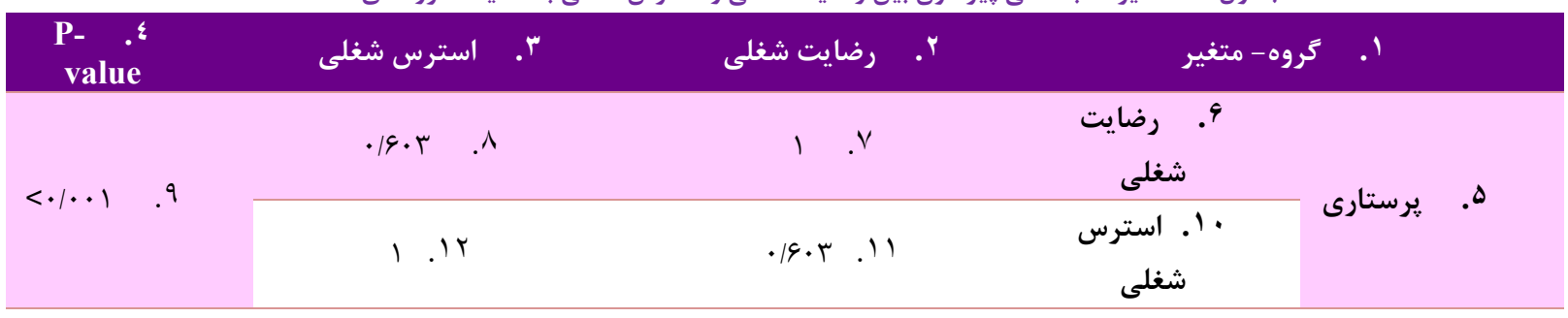




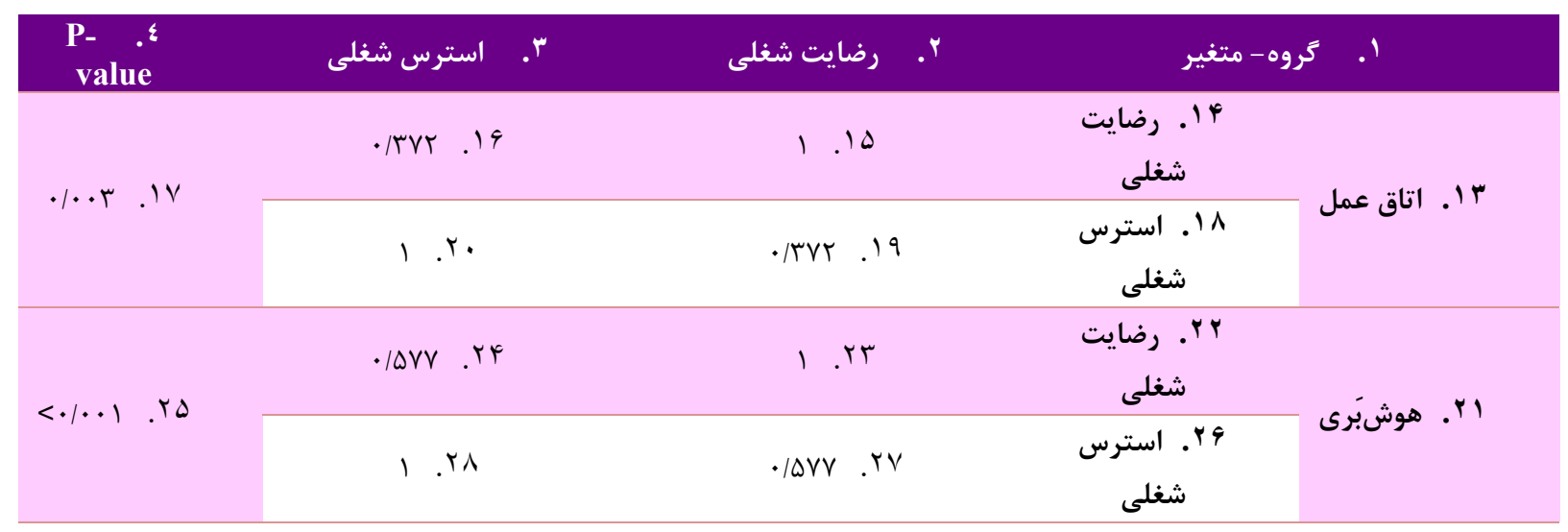

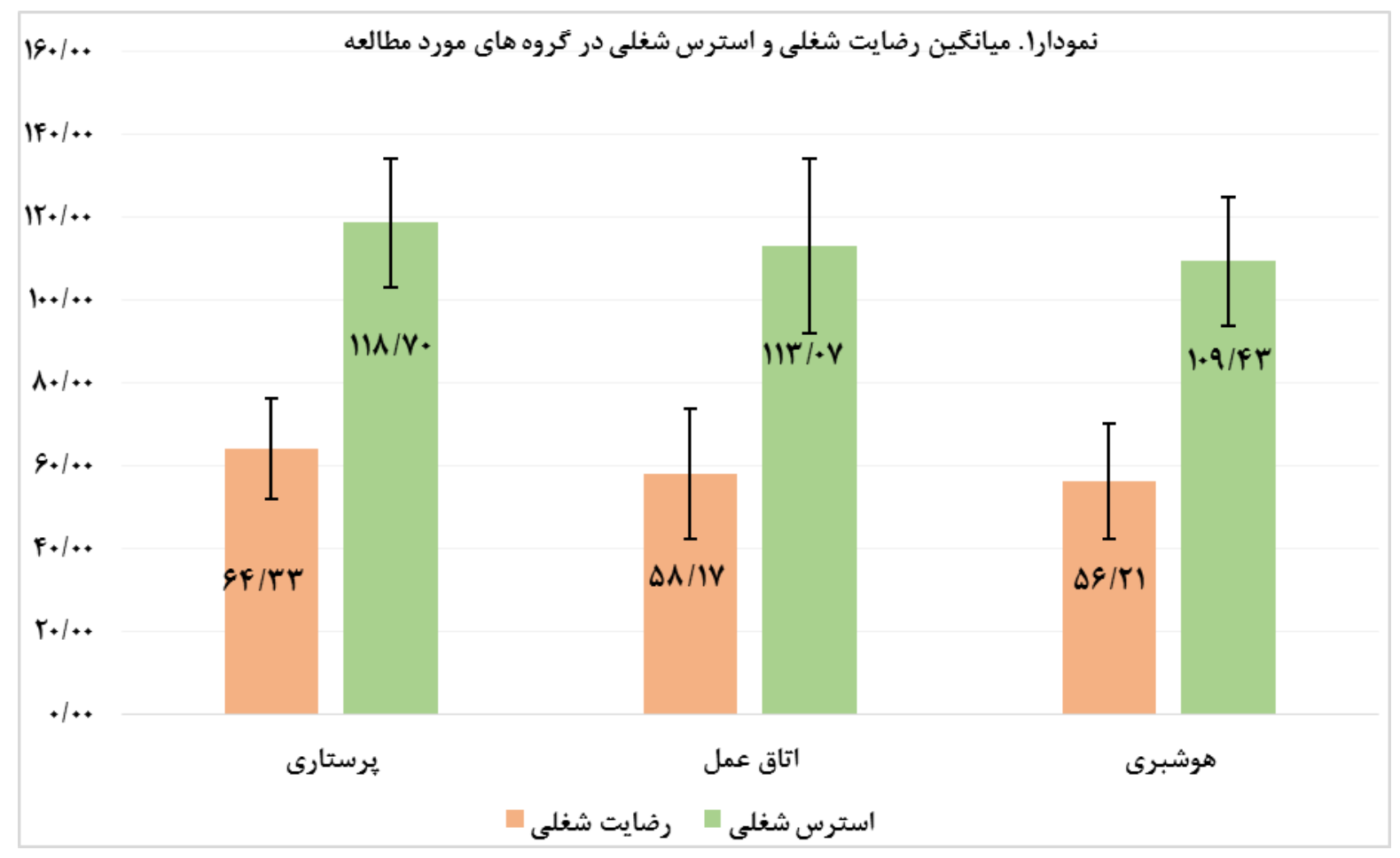

شكل ا. ميانغَين رضايت شغلى و استرس شغلى در تروههاى مطالعهشده

براساس يافتههاى اين مطالعه، در زمينأ رضايت شغلى مشخص شد كه يرستاران بيشترين ميزان رضايت شغلى و وكائ

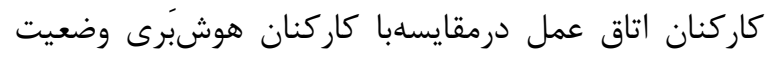

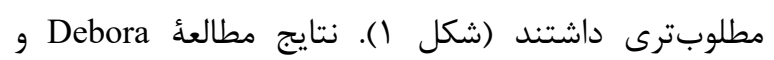
همكاران [عr] نشان داد كه رضايت شغلى كاركنان اتاق عمل

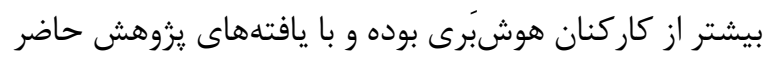

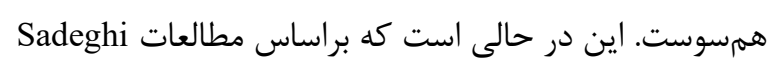

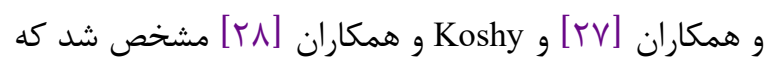
رضايت شغلى كاركنان هوشبَرى از كاركنان اتاق عمل بيشتر

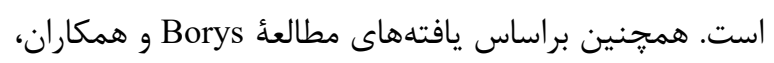
تفاوت معنادارى بين رضايت شغلى كاركنان اتاق عمل و
نيروى انسانى در محيط كار ركنى اصلى است و توجه به

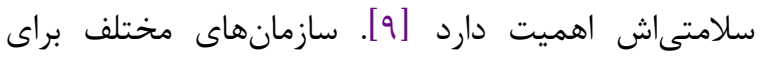
افزايش بهرهورىشان توجه به برخى از مؤلفههاى سازمانى

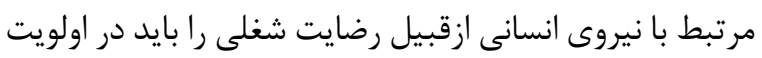
قرار دهند [سب]. باتوجهبه اينكه مطالعات مختلف از اهيطيت آنميت استرس شغلى و تأثير نامطلوب آن بر فرد و سازمان كفتهاند

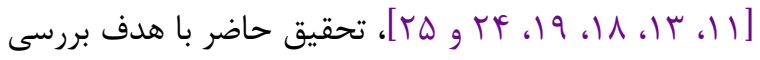
ميزان رضايت شغلى و استرس شغلى در يرستاران و كاركنان

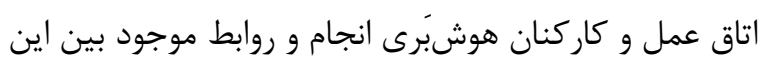
دو مؤلفه بررسى شد. 
مؤثر مانند ميزان درآمد و بُعد خانوار و مؤلفههايى مثل حمايت

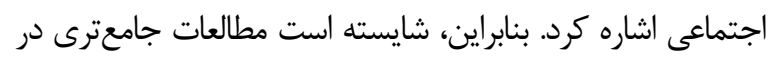

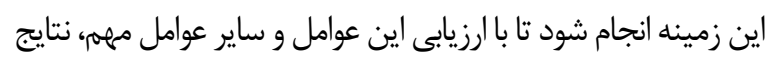

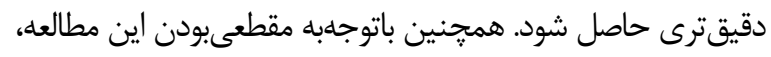

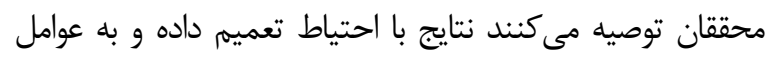
مداخلهكَ ذكرشده در اين بند و نيز ساير مداخلهمَ ها توجه شود.

\section{نتيجه}

براساس نتايج اين مطالعه، در يرسشنامة رضايت شغلى اكثر شركتكنندكان نمرهاى نزديك به ميانكَين كسب كردند.

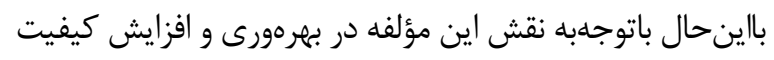
ارائٔ خدمات كاركنان خدمات بهداشتى درمانى، شايسته است

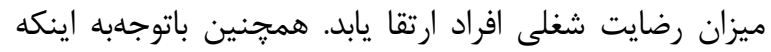

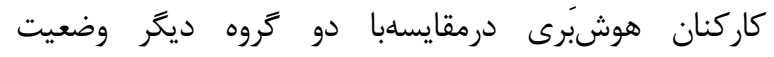

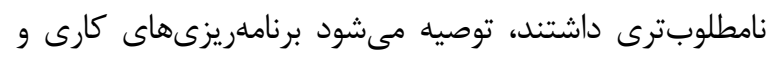
مديريتى در بيمارستانها بهتفكيك تروههاى شغلى انجام شود.

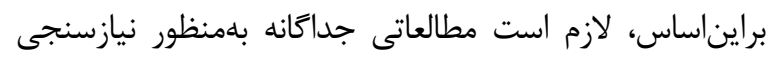

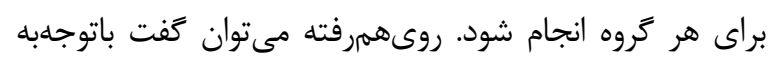
رابطؤ معكوس بين رضايت شغلى و استرس شغلى، با شناسايى عوامل استرسزاى شغلى مىتوان در زمينهُ كاهش ميزان استرس

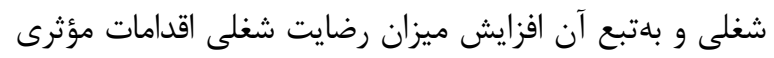

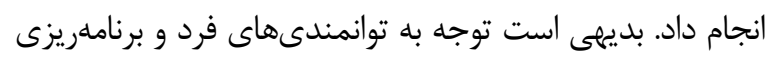
مديريتى مانند كاهش فشار كارى و تنظيم خرخههاى كار -

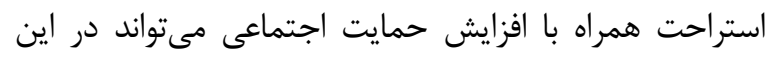
زمينه مؤثر واقع شود.

\section{تقدير و تشكر}

مطالعة حاضر حاصل طرح تحقيقاتى دانشجويى است كه

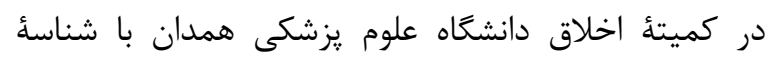

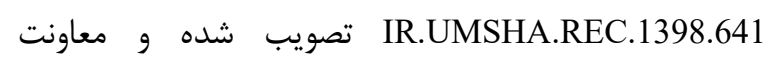
تحقيقات و فناورى اين دانشخاه از آن حمايت كرده است.

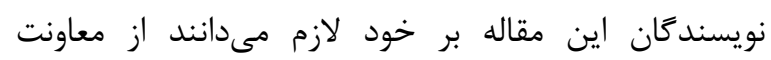

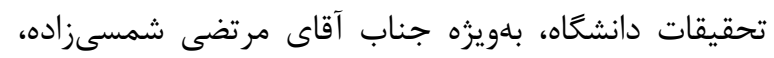

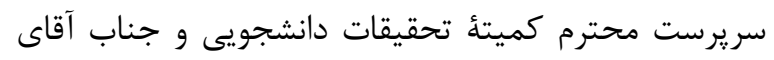
دكتر محمد باباميرى، بابت راهنمايىهاى مفيدشان تقدير و و

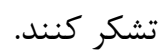

يرستاران شاغل در بخش ICU مشاهده نشد [9]. اين

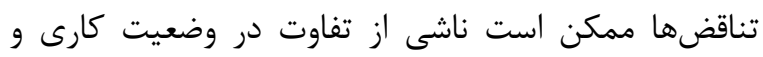

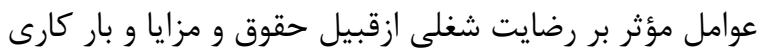

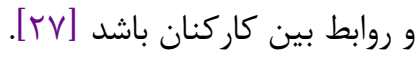

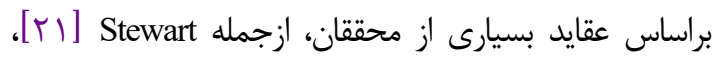
عوامل مختلفى در رضايت شغلى كار كنان مؤثر هستند كه برخى از از إنمان

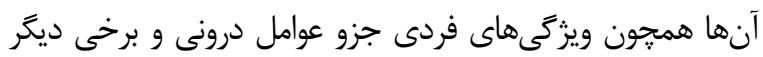

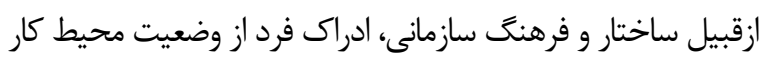
و امنيت شغلى، روابط بين همكاران و رابطه با سريرست يا مافوق

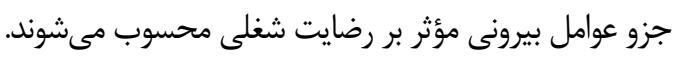

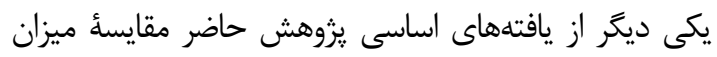
استرس شغلى در كروههاى مطالعهشده بود. در اين زمينه، نتايج

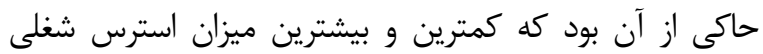

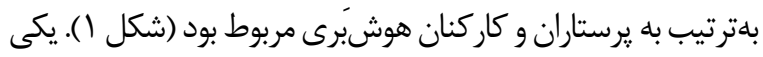

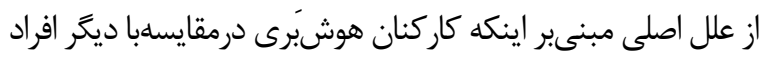
مطالعه استرس بيشترى تجربه مى كنند، ممكن است حساسبوني

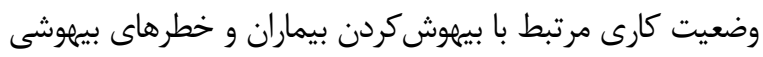

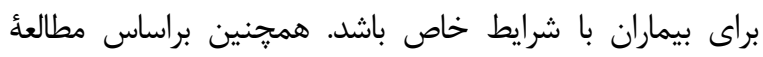

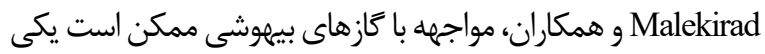
از دلايل بيشتربودن ميزان استرس در كاركنان هوشَبرى و كاركنان

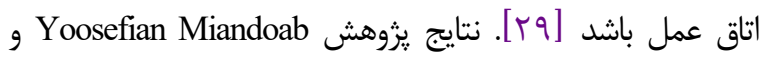
همكاران نيز نشان داد كه كاركنان هوشبَرى بيشتر از كاركنان اتاق

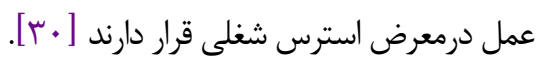

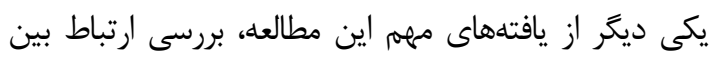

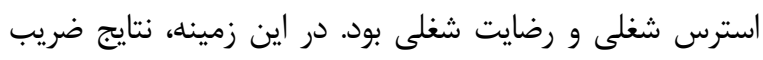
همبستگى ييرسون نشان داد كه بين اين دو مؤلفه سازمانى ازلحاظ

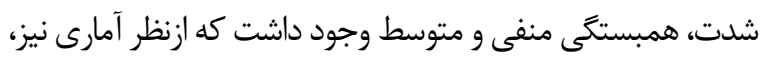

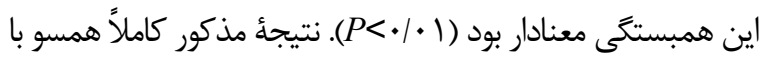

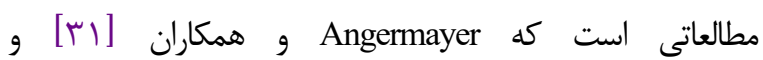
Ghaseminezhad و همكاران [ץ؟] در اين زمينه انجام دادهاند. بلبيانِبهتر، هرجه افراد رضايت شغلى بيشترى داشته باشند، استرس شغلى كمترى نيز تجربه خواهند كرد يا مىتوان اينكونه استنباط

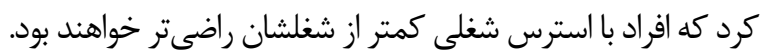
همجنين براساس مطالعه

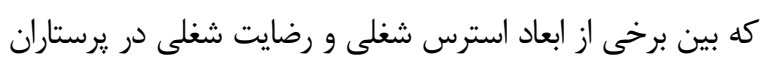
ارتباط معكوس و معنى دارى وجود دارد.

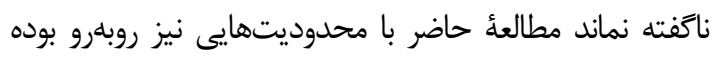

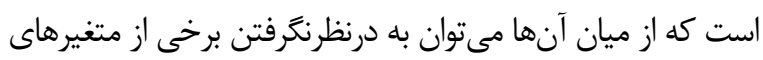




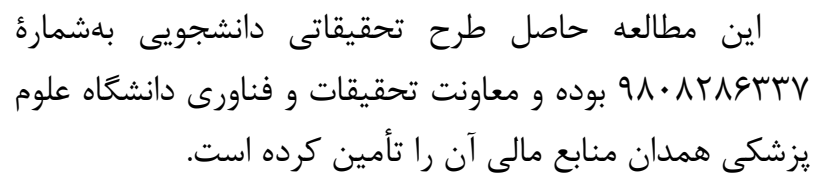

\section{References}

1. Hashemi Habybabady R, Ghaderi N, Rahmani R, Mohammadi M. Estimation of maximum aerobic capacity and its related factors using treadmill test in firefighters of Zahedan. J North Khorasan Uni Med Sci. 2018;10(1):1-8. eng.

2. Rahmani R, Shahnavazi S, Fazli B, Ghasemi F. Ergonomic risk assessment of musculoskeletal disorders in a cement factory workers using QEC technique. Pajouhan Sci J. 2020;18(2):64-72.

3. Ghasemi F, Gholamizadeh K, Doosti-Irani A, Rahmani R. Comparison of Strain Index (SI) and ACGIH-HAL in assessing the risk of upper extremities disorders and prediction of carpal tunnel syndrome in butchers. Iran J Ergon. 2019;6(4):1-8. [DOI:10.30699/jergon.6.4.1]

4. Ghasemi F, Samavat P, Soleimani F. The links among workload, sleep quality, and fatigue in nurses: A structural equation modeling approach. Fatigue. 2019;7(3):141-52. [DOI:10.1080/21641846.2019.1652422]

5. Akbari H, Ghasemi F, Akbari H, Adibzadeh A. Predicting needlestick and sharps injuries and determining preventive strategies using a Bayesian network approach in Tehran, Iran. Epidemiol Health. 2018;40:e2018042］[DOI:10.4178/epih.e2018042] [PMID] [PMCID]

6. Almasi Z, Hashemi Habib-abadi R, Rahmani R. The study of mental health status and its effective factors among Zahedan city's welders. Pajouhan Sci J. 2019;18(2):1-7.

7. De Cieri H, Shea T, Cooper B, Oldenburg B. Effects of work-related stressors and mindfulness on mental and physical health among Australian nurses and healthcare workers. J Nurs Scholarsh. 2019;51(5):580-89. [DOI:10.1111/jnu. 12502] [PMID]

8. Noorian C, Parvin N, Mehrabi T. Evaluation of the relationship between occupational stress and general health condition in nurses working in Isfahan university hospitals. J Nurs Midwifery Rafsanjan. 2005;5(1, 2):45-52.

9. Borys M, Wiech M, Zyzak K, Majchrzak A, Kosztyła A, Michalak A, et al. Job satisfaction among anesthetic and intensive care nurses-multicenter, observational study. Anaesthesiol Intensive Ther. 2019;51(2):102-6. [DOI:10.5114/ait.2019.85804] [PMID]

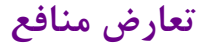 \\ بين نويسندكان هيجَّونه تعارضى در منافع وجود ندارد.

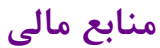

10. Yousefi S, Nayebzadeh S, Eslami H. The effects of job stress on accountants job satisfaction. Iran Occup Health. 2015;12(3):46-53.

11. Yaacob M, Long CS. Role of occupational stress on job satisfaction. Mediterranean J Soc Sci. 2015;6(2 S1):81-87. [DOI:10.5901/mjss.2015.v6n2s1p81]

12. Rajaei M, Haghighi H, Dadipoor S, Fallaghi S, Salimi M, SareghiBrojeni M, et al. Relationship between occupational stress and job satisfaction among Iranian obstetricians. Reposit Res Invest Inform. 2013;17(3):249-55.

13. Ghafourian H, Ghasemi I, Ebrahimi M. The impact of job stress on job satisfaction among school administrators. J Modern Thoughts Edu. 2011;6(4):33-48.

14. Khamisa N, Oldenburg B, Peltzer K, Ilic D. Work related stress, burnout, job satisfaction and general health of nurses. Int J Environ Res Public Health. 2015;12(1):652-66. [DOI:10.3390/ijerph120100652] [PMID] [PMCID]

15. Ansari H, Fazli B, Zare H, Hami Mahkoyeh S, Fazli F. Job stress and its relationship with the musculoskeletal disorders among office workers of Zahedan university of medical sciences, Iran. Iran J Health Sci. 2016;4(1):10-19. [DOI:10.18869/acadpub.jhs.4.1.10]

16. Babamiri M, Zahiri A, Neissi A, Arshadi N, Shahroee S. Job Stressors as predictors of psychosomatic symptoms. J Ilam Uni Med Sci. 2015;23(1):45-55.

17. Sadeghi A, Goharloo Arkawaz A, Cheraghi F, Moghimbeigi A. Relationship between head nurses' servant leadership style and nurses' job satisfaction. Q J Nurs Manag. 2015;4(1):28-38.

18. Mohammadi SZ, Haghighi SA. Relation between job stress and burnout among nursing staff. Avicenna J Nurs Midwifery Care. 2011;19(2):42-52.

19. Biganeh J, Abolghasemi J, Alimohammadi I, Ebrahimi H, Torabi Z, Ashtarinezhad A. Survey of occupational stress effects on burnout among nurses. J Knowl Health. 2018;13(1):10-18.

20. Brayfield AH, Rothe HF. An index of job satisfaction. J Appl Psychol. 1951;35(5):307. [DOI: $10.1037 / \mathrm{h} 0055617$ ]

21. Hassani M, Sedaqat R, Kazemzadehbeytali M. Correlation between the Ethical climate, Job stress 
and Job Satisfaction in Nurses. Med Ethics J. 2017;11(40):63-71. [DOI:10.21859/mej-114063]

22. Azad Marzabadi E, Gholami FM. Reliability and validity assessment for the HSE job stress questionnaire. Int J Behav Sci. 2011;4(4):291-97.

23. Mosadeghrad AM. Occupational stress and turnover intention: implications for nursing management. Int $\mathrm{J}$ Health Policy Manag. 2013;1(2):169. [DOI:10.15171/ijhpm.2013.30] [PMID] [PMCID]

24. Caulfield N, Chang D, Dollard MF, Elshaug C. A Review of Occupational Stress Interventions in Australia. International Journal of Stress Manag. 2004;11(2):149-166.

[DOI:10.1037/10725245.11.2.149]

25. Daniali S. Job satisfaction and job stress among staff of health center. 2014.

26. A D, J G, R D, K L. Job satisfaction in the operating room. UMI 2008;13(2):1-109.

27. Sadeghi S, Sabour B, Azizi Y, Tohidnia MR, Moeenian KS, Hoseini J. A comparative study of job satisfaction and related factors in anesthesia and operating room staffs of Kermanshah university of medical science (2012). 2014.

28. Koshy RC, Ramesh B, Khan S, Sivaramakrishnan A. Job satisfaction and stress levels among anaesthesiologists of south India. Indian J Anaesth. 2011;55(5):513-17. [DOI:10.4103/0019-5049.89891] [PMID] [PMCID]
29. Malekirad AA, Ranjbar A, Rahzani K, Kadkhodaee M, Rezaie A, Taghavi B, et al. Oxidative stress in operating room personnel: Occupational exposure to anesthetic gases. Hum Exp Toxicol. 2005;24(11):597601. [DOI:10.1191/0960327105ht565oa] [PMID]

30. Yoosefian Miandoab N, Charkhat Gorgich E, Rezvani Amin M, Shahrakipoor M. Occupational stress in operating room and anesthesia technicians of Zahedan Ali ebneAbitaleb Hospital. Beyhagh. 2015;20(2):3341.

31. Angermeyer MC, Bull N, Bernert S, Dietrich S, Kopf A. Burnout of caregivers: A comparison between partners of psychiatric patients and nurses. Arch Psychiatr Nurs. 2006;20(4):158-65. [DOI:10.1016/j.apnu.2005.12.004] [PMID]

32. Ghaseminezhad A, Siadat SA, Nouri A. A study of the relationship between organizational climate and teachers'job stress and job satisfaction. 2005.

33. Bartram T, Joiner TA, Stanton P. Factors affecting the job stress and job satisfaction of Australian nurses: Implications for recruitment and retention. Contemp Nurse. 2004;17(3):293-304. [DOI:10.5172/conu.17.3.293] [PMID] 